\title{
Pseudospherical functions on a hyperboloid of one sheet
}

\author{
K Kowalski, J Rembieliński and A Szcześniak \\ Department of Theoretical Physics, University of Łódź, ul. Pomorska 149/153, \\ 90-236 Łódź, Poland
}

\begin{abstract}
The pseudospherical functions on one-sheet, two-dimensional hyperboloid are discussed. The simplest method of construction of these functions is introduced using the Fock space structure of the representation space of the $s u(1,1)$ algebra. The pseudospherical functions with half-integer order are investigated. The counterparts of the Legendre functions for the hyperboloid are introduced and a new class of pseudospherical functions is found.
\end{abstract}

PACS numbers: 02.20.Sv, 02.30.Gp, 02.40.-k, 03.65.-w, 04.20.Cv

\section{Introduction}

The one-sheet hyperboloid is an important object in both general relativity and quantum mechanics. Indeed, on the one hand, it is a model of the two-dimensional spacetime with constant curvature (de Sitter space). Furthermore, its invariance group $S O(2,1)$ plays the fundamental role in the study of representations of the $(2+1)$ - Poincaré group and corresponding relativistic wave equations [1]. Besides of the elementary particle physics the range of applications of the $S O(2,1)$ group include quantum optics where this group is applied in the theory of coherent states, especially the squeezed states [2], and the classical optics [3]. The quantum systems related to the one-sheet hyperboloid were discussed in the papers [4-10]. In particular, Dane and Vardiyev [4] studied Schrödinger equations with Pöschl-Teller like potentials connected with one and two-sheet hyperboloid and found the explicit expressions of the Green's functions of a free particle on those spaces. The contraction of eigenfunctions for the Laplace equation on hyperboloid of one sheet and pseudoeuclidean space have been considered in the paper of Pogosyan, Sissakian and Winternitz [5]. The quantization of particle dynamics on one-sheet hyperboloid embedded in three-dimensional Minkowski space was discussed by Piechocki and Jorjadze in papers [6, 7, 8]. The coherent state quantization of particle in the two-dimensional de Sitter space i.e. one-sheet hyperboloid, was investigated by Gazeau and Piechocki in [9]. 
The pseudospherical functions in the general case of hyperboloids of arbitrary dimensions were introduced independently, using different methods by Ra̧czka, Limić and Niederle [11] and Strichartz [12]. Both approaches are based on the very general mathematical scheme involving multidimensional hyperboloids, no wonder that the physically interesting case of the two-dimensional hyperboloid of one sheet was not discussed therein in a more detail. Recently, the wavefunctions for the two-dimensional hyperboloids involving their different parametrizations were studied in [4]. The approach used in [4] for the identification of wavefunctions in the hyperbolic parametrization of hyperboloids coincide with that adopted in [11].

In this work we construct the pseudospherical functions for the quantum mechanics on a one-sheet hyperboloid using the simplest algorithm developed for the case of the sphere $S^{2}$, based on application of the Fock space structure of representations of the $S O(2,1)$ group. The approach taken up in this work is more general than alternative ones mentioned earlier because it enables fixing the phase of the pseudospherical functions. Surprisingly, to our knowledge, despite the fact that the pseudospherical functions for hyperboloids are also referred to as the "spherical functions" or "spherical harmonics" (see for example [13]) such natural approach was not taken up so far. Utilizing the introduced formalism we study for the first time in the literature the pseudospherical functions with half-integer order. We also identify the pseudospherical counterparts of the Legendre functions and study their basic properties in the case of the discrete series representations. Finally, we find a new class of pseudospherical functions related to the discrete series.

\section{Basic representations}

We first discuss the basic properties of representations of the $S O(2,1)$ group which is the invariance group for the one-sheet hyperboloid such that

$$
\left(x^{1}\right)^{2}+\left(x^{2}\right)^{2}-\left(x^{3}\right)^{2}=a^{2},
$$

where $a>0$ is a parameter. The generators $K_{i}, i=1,2,3$, of the representations of $S O(2,1)$ satisfy the following commutation relations:

$$
\left[K_{3}, K_{\alpha}\right]=\mathrm{i} \varepsilon_{\alpha \beta} K_{\beta}, \quad\left[K_{\alpha}, K_{\beta}\right]=-\mathrm{i} \varepsilon_{\alpha \beta} K_{3}, \quad \alpha, \beta=1,2 .
$$

The algebra $s o(2,1)(2.2)$ expressed with the help of operators $K_{3}, K_{ \pm}=K_{1} \pm \mathrm{i} K_{2}$ takes the form

$$
\left[K_{3}, K_{ \pm}\right]= \pm K_{ \pm}, \quad\left[K_{+}, K_{-}\right]=-2 K_{3}
$$

The Casimir operator of the algebra (2.3) is given by

$$
C_{2}=K_{3}^{2}-K_{1}^{2}-K_{2}^{2}=K_{3}\left(K_{3}+1\right)-K_{-} K_{+}=K_{3}\left(K_{3}-1\right)-K_{+} K_{-} .
$$

Consider the unitary irreducible representation of the algebra (2.3) spanned by the (normalized) common eigenvectors $|k, m\rangle$ of the operators $C_{2}$ and $K_{3}$ :

$$
C_{2}|k, m\rangle=k(k+1)|k, m\rangle, \quad K_{3}|k, m\rangle=m|k, m\rangle .
$$


Recall that 14

$$
\begin{aligned}
& K_{+}|k, m\rangle=\sqrt{(m-k)(m+k+1)}|k, m+1\rangle, \\
& K_{-}|k, m\rangle=\sqrt{(m+k)(m-k-1)}|k, m-1\rangle .
\end{aligned}
$$

The unitary irreducible representations can be grouped into three classes accordingly to the spectrum of $C_{2}$ and $K_{3}$. The discrete series $D_{+}$and $D_{-}$. The series $D_{+}$is specified by

$$
k=-\frac{1}{2}, 0, \frac{1}{2}, 1, \ldots, \quad m \geq k+1 .
$$

This series is bounded below. Namely

$$
K_{-}|k, k+1\rangle=0 .
$$

Using (2.6a) we find that the vectors $|k, m\rangle$ can be obtained from the lowest-weight state $|k, k+1\rangle$ via

$$
|k, m\rangle=\sqrt{\frac{(2 k+1) !}{(m-k-1) !(m+k) !}} K_{+}^{m-k-1}|k, k+1\rangle, \quad m \geq k+1 .
$$

For the discrete series $D_{-}$we have

$$
k=-\frac{1}{2}, 0, \frac{1}{2}, 1, \ldots, \quad m \leq-(k+1) .
$$

The series $D \_$is bounded above. Namely

$$
K_{+}|k,-(k+1)\rangle=0 .
$$

The counterpart of $(2.9)$ is

$$
|k, m\rangle=\sqrt{\frac{(2 k+1) !}{[-(m+k+1)] !(k-m) !}} K_{-}^{-(m+k+1)}|k,-(k+1)\rangle, \quad m \leq-(k+1) .
$$

The second class is the continuous principal series such that

$$
k=-\frac{1}{2}+\mathrm{i} \lambda, \quad \lambda>0, \quad m=0, \pm 1, \pm 2, \ldots \text { or } m= \pm \frac{1}{2}, \pm \frac{3}{2}, \ldots
$$

Finally, we have the continuous supplementary series

$$
-\frac{1}{2}<k<0, \quad m=0, \pm 1, \pm 2, \ldots
$$

Bearing in mind the possible applications we remark that the discrete series representations of $S U(1,1)$ which is locally isomorphic to $S O(2,1)$, were related in [15] to bound states and the continuous series representations to the scattering states.

We now discuss the realization of generators by means of the differential operators. We first write down the following realization of the algebra (2.2):

$$
\begin{aligned}
& K_{1}=-\mathrm{i}\left(x^{2} \partial_{3}+x^{3} \partial_{2}\right), \\
& K_{2}=\mathrm{i}\left(x^{1} \partial_{3}+x^{3} \partial_{1}\right), \\
& K_{3}=-\mathrm{i}\left(x^{1} \partial_{2}-x^{2} \partial_{1}\right),
\end{aligned}
$$


where $\partial_{i} \equiv \partial / \partial x^{i}, i=1,2,3$. Introducing the polar hyperbolic coordinates (biharmonic coordinates) such that

$$
\begin{aligned}
& x^{1}=a \cosh \tau \cos \varphi, \\
& x^{2}=a \cosh \tau \sin \varphi, \\
& x^{3}=a \sinh \tau,
\end{aligned}
$$

so the hyperboloid (2.1) is specified by $a=$ const, $\tau \in \mathbb{R}$, and $\varphi \in[0,2 \pi)$, we get from $(2.15)$

$$
\begin{aligned}
& K_{+}=-e^{i \varphi}\left(\frac{\partial}{\partial \tau}+\operatorname{itgh} \tau \frac{\partial}{\partial \varphi}\right), \\
& K_{-}=e^{-i \varphi}\left(\frac{\partial}{\partial \tau}-\operatorname{itgh} \tau \frac{\partial}{\partial \varphi}\right) \\
& K_{3}=-\mathrm{i} \frac{\partial}{\partial \varphi}
\end{aligned}
$$

Hence

$$
C_{2}=\frac{\partial^{2}}{\partial \tau^{2}}+\operatorname{tgh} \tau \frac{\partial}{\partial \tau}-\frac{1}{\cosh ^{2} \tau} \frac{\partial^{2}}{\partial \varphi^{2}}
$$

Clearly, the pseudospherical functions $\mathcal{Y}_{k}^{m}(\tau, \varphi)$, i.e. the realizations of the abstract vectors $|k, m\rangle$ in the coordinate representations, satisfy the system of differential equations

$$
\begin{aligned}
& C_{2} \mathcal{Y}_{k}^{m}(\tau, \varphi)=k(k+1) \mathcal{Y}_{k}^{m}(\tau, \varphi), \\
& K_{3} \mathcal{Y}_{k}^{m}(\tau, \varphi)=m \mathcal{Y}_{k}^{m}(\tau, \varphi),
\end{aligned}
$$

where $C_{2}$ and $K_{3}$ are given by $(2.18)$ and $(2.17 \mathrm{c})$, respectively. Separating variables we find that $\mathcal{Y}_{k}^{m}(\tau, \varphi)$ is of the form

$$
\mathcal{Y}_{k}^{m}(\tau, \varphi)=e^{\mathrm{i} m \varphi} f_{k}^{m}(\tau)
$$

where $f_{k}^{m}(\tau)$ fulfils

$$
\frac{d^{2} f_{k}^{m}(\tau)}{d \tau^{2}}+\operatorname{tgh} \tau \frac{d f_{k}^{m}(\tau)}{d \tau}+\left[-k(k+1)+\frac{m^{2}}{\cosh ^{2} \tau}\right] f_{k}^{m}(\tau)=0 .
$$

In other words, the system (2.19) can be brought down to eq. (2.21).

\section{Pseudospherical functions for discrete series representations}

We now discuss the pseudospherical functions $\mathcal{Y}_{k}^{m}(\tau, \varphi)$ in the case of the discrete series $D_{+}$. The equation (2.21) was the point of departure for finding pseudospherical functions in [11]. The solution to (2.21) was actually guessed in [11] (see (3.17) below). We now apply the simpler and most natural method for constructing the pseudospherical functions based on the well-known approach taken up for spherical functions (see for example [16]). Consider the case of the series $D_{+}$. By the last equation of (2.4) the wavefunction

$$
\mathcal{Y}_{k}^{k+1}(\tau, \varphi)=e^{\mathrm{i}(k+1) \varphi} f_{k}^{k+1}(\tau)
$$


representing the lowest-weight state $|k, k+1\rangle$, satisfying

$$
K_{-} \mathcal{Y}_{k}^{k+1}(\tau, \varphi)=0
$$

is the solution of the system (2.19) with $m=k+1$. Using (2.17b) we get from (3.2) the first order equation

$$
\frac{d f_{k}^{k+1}(\tau)}{d \tau}+(k+1) \operatorname{tgh} \tau f_{k}^{k+1}(\tau)=0
$$

The solution of the elementary equation (3.3) is

$$
f_{k}^{k+1}(\tau)=c_{k} \cosh ^{-(k+1)} \tau,
$$

where $c_{k}$ is a normalization constant. Now, the Hilbert space of square integrable functions on a hyperboloid of one sheet (2.1) is specified by the scalar product

$$
\langle F \mid G\rangle=\int_{-\infty}^{\infty} \cosh \tau d \tau \int_{0}^{2 \pi} d \varphi F^{*}(\tau, \varphi) G(\tau, \varphi),
$$

where we set without loose of generality $a=1$. Hence, using the identity [17]

$$
\int_{0}^{\infty} \frac{d x}{\cosh ^{2 k+1} x}=\frac{(2 k-1) ! !}{(2 k) ! !} \frac{\pi}{2}
$$

where $k=0,1,2, \ldots$, we get

$$
c_{k}=\frac{1}{\sqrt{2} \pi} \sqrt{\frac{(2 k) ! !}{(2 k-1) ! !}}=\frac{2^{k-\frac{1}{2}}}{\pi} \frac{k !}{\sqrt{(2 k) !}} .
$$

Furthermore, taking into account (2.17a) we obtain the identity

$$
K_{+}^{r}\left[e^{\mathrm{i} m \varphi} f(\tau)\right]=(-1)^{r} e^{\mathrm{i}(m+r) \varphi}\left(\cosh ^{m+r} \tau \frac{d^{r}}{d(\sinh \tau)^{r}} \cosh ^{-m} \tau\right) f(\tau) .
$$

An immediate consequence of (2.9), (3.8), (3.1), (3.4) and (3.7) is the following formula on the pseudospherical functions $\mathcal{Y}_{k}^{m}$ :

$\mathcal{Y}_{k}^{m}(\tau, \varphi)=(-1)^{m-k-1} 2^{k} k ! \sqrt{\frac{2 k+1}{2 \pi^{2}(m-k-1) !(m+k) !}} e^{\mathrm{i} m \varphi} \cosh ^{m} \tau \frac{d^{m-k-1}}{d(\sinh \tau)^{m-k-1}} \cosh ^{-2(k+1)} \tau$

The relation (3.9) can be written as

$$
\mathcal{Y}_{k}^{m}(\tau, \varphi)=\sqrt{\frac{2 k+1}{2 \pi^{2}(m-k-1) !(m+k) !}} e^{\mathrm{i} m \varphi} \mathcal{P}_{k}^{m}(\sinh \tau),
$$

where $\mathcal{P}_{k}^{m}(x)$ are counterparts of the (associated) Legendre functions connected with spherical harmonics, defined by

$$
\mathcal{P}_{k}^{m}(x)=(-1)^{m-k-1} 2^{k} k !\left(1+x^{2}\right)^{\frac{m}{2}} \frac{d^{m-k-1}}{d x^{m-k-1}} \frac{1}{\left(1+x^{2}\right)^{k+1}}
$$


The basic recurrences satisfied by the functions $\mathcal{P}_{k}^{m}$ analogous to the well-known formulas on the associated Legendre polynomials are introduced in Appendix. Now taking into account the identity [18]

$$
\frac{d^{n}}{d x^{n}} f\left(x^{2}\right)=n ! \sum_{r=0}^{\left[\frac{n}{2}\right]} \frac{(2 x)^{n-2 r}}{r !(n-2 r) !} f^{(n-r)}\left(x^{2}\right),
$$

where $[p]$ is the biggest integer in $p$, we find

$$
\mathcal{P}_{k}^{m}(x)=2^{k}(m-k-1) !\left(1+x^{2}\right)^{\frac{m}{2}} \sum_{r=0}^{\left[\frac{m-k-1}{2}\right]}(-1)^{r} \frac{(m-r-1) !}{r !(m-k-1-2 r) !} \frac{(2 x)^{m-k-1-2 r}}{\left(1+x^{2}\right)^{m-r}}
$$

which leads to

$\mathcal{P}_{k}^{m}(\sinh \tau)=2^{k}(m-k-1) ! \cosh ^{-(k+1)} \tau \sum_{r=0}^{\left[\frac{m-k-1}{2}\right]}(-1)^{r} \frac{(m-r-1) !}{r !(m-k-1-2 r) !}(2 \operatorname{tgh} \tau)^{m-k-1-2 r}$

Hence, using (3.10) the pseudospherical functions can be written in explicit form as

$$
\begin{aligned}
\mathcal{Y}_{k}^{m}(\tau, \varphi)= & 2^{k} \sqrt{\frac{(2 k+1)(m-k-1) !}{2 \pi^{2}(m+k) !}} e^{\mathrm{i} m \varphi} \cosh ^{-(k+1)} \tau \\
& \times \sum_{r=0}^{\left[\frac{m-k-1}{2}\right]}(-1)^{r} \frac{(m-r-1) !}{r !(m-k-1-2 r) !}(2 \operatorname{tgh} \tau)^{m-k-1-2 r} .
\end{aligned}
$$

The authors did not find the formula on the pseudospherical functions of the form (3.15) in the literature. The sum from $(3.15)$ and thus $\mathcal{Y}_{k}^{m}(\tau, \varphi)$ can be expressed in terms of the hypergeometric functions ${ }_{2} F_{1}(\alpha, \beta ; \gamma ; z)$. Namely, we find after some calculations

$$
\begin{aligned}
\mathcal{Y}_{k}^{m}(\tau, \varphi)= & (-1)^{\frac{1}{2}(m-k-1)} \sqrt{\frac{(2 k+1) \Gamma\left(\frac{1}{2}(m-k)\right) \Gamma\left(\frac{1}{2}(m+k+1)\right)}{4 \pi^{2} \Gamma\left(\frac{1}{2}(m-k+1)\right) \Gamma\left(\frac{1}{2}(m+k+2)\right)}} e^{\mathrm{i} m \varphi} \cosh ^{-(k+1)} \tau \\
& \times{ }_{2} F_{1}\left[\frac{1}{2}(m+k+1), \frac{1}{2}(-m+k+1) ; \frac{1}{2} ; \operatorname{tgh}^{2} \tau\right]
\end{aligned}
$$

where $m-k=2 n+1, n=0,1,2, \ldots, \Gamma(x)$ is the gamma function, and

$$
\begin{aligned}
\mathcal{Y}_{k}^{m}(\tau, \varphi)= & (-1)^{\frac{1}{2}(m-k-2)} \sqrt{\frac{(2 k+1) \Gamma\left(\frac{1}{2}(m-k+1)\right) \Gamma\left(\frac{1}{2}(m+k+2)\right)}{\pi^{2} \Gamma\left(\frac{1}{2}(m-k)\right) \Gamma\left(\frac{1}{2}(m+k+1)\right)}} e^{\mathrm{i} m \varphi} \cosh ^{-(k+1)} \tau \operatorname{tgh} \tau \\
& \times{ }_{2} F_{1}\left[\frac{1}{2}(m+k+2), \frac{1}{2}(-m+k+2) ; \frac{3}{2} ; \operatorname{tgh}^{2} \tau\right]
\end{aligned}
$$

where $m-k=2 n+2$ and $n=0,1,2, \ldots$ Up to the absent phase factor $(-1)^{\frac{1}{2}(m-k-1)}$ in (3.16a) and the phase factor $(-1)^{\frac{1}{2}(m-k-2)}$ in $(3.16 \mathrm{~b})$ as well as some typo in the formula referring to (3.16b) the solution (3.16) was guessed from (2.21) by Raczka, Limić and Niederle [11] and rediscovered by Dane and Verdiyev [4]. More precisely, one can put

$$
f_{k}^{m}(\tau)=\cosh ^{\alpha} \tau g_{k}^{m}(\tau)
$$


which leads to

$\frac{d^{2} g_{k}^{m}(\tau)}{d \tau^{2}}+(2 \alpha+1) \operatorname{tgh} \tau \frac{d g_{k}^{m}(\tau)}{d \tau}+\left[\alpha(\alpha+1)-k(k+1)+\frac{m^{2}-\alpha^{2}}{\cosh ^{2} \tau}\right] g_{k}^{m}(\tau)=0$

Hence setting $\alpha=-(k+1)$ and making the ansatz

$$
g_{k}^{m}(\tau)=h_{k}^{m}\left(\operatorname{tgh}^{2} \tau\right)
$$

we obtain the hypergeometric equation

$x(1-x) h_{k}^{m \prime \prime}(x)+\left[\frac{1}{2}-(k+2) x\right] h_{k}^{m \prime}(x)+\frac{1}{4}\left[m^{2}-(k+1)^{2}\right] h_{k}^{m}(x)=0$

satisfied by the hypergeometric function ${ }_{2} F_{1}\left[\frac{1}{2}(m+k+1), \frac{1}{2}(-m+k+1) ; \frac{1}{2} ; x\right]$. As is well known if ${ }_{2} F_{1}(\alpha, \beta ; \gamma ; z)$ is a solution of the hypergeometric equation and $\gamma$ is not integer, then the second linearly independent solution is $z^{1-\gamma}{ }_{2} F_{1}(\alpha-\gamma+1, \beta-\gamma+1 ; 2-\gamma ; z)$. Therefore, the second linearly independent solution to $(3.20)$ is $\sqrt{x}_{2} F_{1}\left[\frac{1}{2}(m+k+\right.$ $\left.2), \frac{1}{2}(-m+k+2) ; \frac{3}{2} ; x\right]$. On putting in the both solutions $x=\operatorname{tgh}^{2} \tau$ and using (3.17), (3.19) and (2.20) we arrive, up to the normalization constant, at the solutions (3.16a) and (3.16b), respectively. We point out that the problem of normalization of solutions to (3.20) is a nontrivial task based on utilization of some identities satisfied by hypergeometric functions (compare [4]). On the other hand, the normalization of pseudospherical functions in our approach is ensured by normalization of the elementary function (3.4).

We stress that the phase factor in (3.16a) and (3.16b) is not arbitrary and its correct form ensures that $K_{+}$and $K_{-}$act on functions $\mathcal{Y}_{k}^{m}(\tau, \varphi)$ as ladder operators in accordance with (2.6). Clearly, the phase cannot be fixed based only on eq. (2.21).

The possibility of generation of pseudospherical functions by the action of the raising operator on the lowest-weight state and lowering operator on the highest-weight state in the case of the series $D_{+}$and $D_{-}$, respectively was recognized in [1]. However, no explicit formulae on the pseudospherical functions in the general case of arbitrary $m \geq k+1\left(\right.$ series $\left.D_{+}\right)$, and $m \leq-(k+1)$ (series $\left.D_{-}\right)$were provided in [1].

As remarked in [1] by making the ansatz

$$
f_{k}^{m}(\tau)=u_{k}^{m}(\mathrm{i} \sinh \tau)
$$

one can reduce (2.21) to the equation of the form

$$
\left(1-z^{2}\right) u_{k}^{m \prime \prime}(z)-2 z u_{k}^{m \prime}(z)+\left[k(k+1)-\frac{m^{2}}{1-z^{2}}\right] u_{k}^{m}(z)=0
$$

satisfied by the associated Legendre functions $P_{k}^{m}(z)$ (spherical harmonics). Nevertheless, it is not clear what is advantage of such reduction. For example, we have $P_{k}^{m}(z) \equiv 0$ for $m \geq k+1$ [21], while this inequality is satisfied by indices of all pseudospherical functions in the case of the representation $D_{+}$. Furthermore, the divergence of the norm of the functions $P_{k}^{m}(i \sinh \tau)$ in the case of the continuous principal series with $k=-\frac{1}{2}+\mathrm{i} \lambda$ was reported in [1].

We finally point out that the counterpart of the relations (3.16) in the case of the spherical harmonics is of the form

$Y_{j}^{m}(\theta, \varphi)=a_{m j} e^{\mathrm{i} m \varphi} \sin ^{j} \theta_{2} F_{1}\left(-\frac{1}{2}(j-m),-\frac{1}{2}(j+m) ; \frac{1}{2} ;-\operatorname{ctg}^{2} \theta\right)$, 
where $j-m=2 n$, and $n=1, \ldots, j ; a_{m j}$ is a normalization constant, and

$Y_{j}^{m}(\theta, \varphi)=b_{m j} e^{\mathrm{i} m \varphi} \sin ^{j} \theta \operatorname{ctg} \theta_{2} F_{1}\left(-\frac{1}{2}(j-m-1),-\frac{1}{2}(j+m-1) ; \frac{3}{2} ;-\operatorname{ctg}^{2} \theta\right)$,

where $j-m=2 n-1, n=1, \ldots, j$, and $b_{m j}$ is a normalization constant.

We now discuss the pseudospherical functions in the case of the series $D_{-}$. Taking into account the second equation of (2.4) we find that the wavefunction

$$
\tilde{\mathcal{Y}}_{k}^{-(k+1)}(\tau, \varphi)=e^{-\mathrm{i}(k+1) \varphi} f_{k}^{-(k+1)}(\tau)
$$

representing the highest-weight state $|k,-(k+1)\rangle$, such that

$$
K_{+} \tilde{\mathcal{Y}}_{k}^{-(k+1)}(\tau, \varphi)=0
$$

is the solution to $(3.5)$, where $m=-(k+1)$. Moreover, it follows immediately from (3.3a) and (3.25) that the function $f_{k}^{-(k+1)}(\tau)$ fulfils the same equation (3.3) as $f_{k}^{k+1}(\tau)$, so we set $f_{k}^{-(k+1)}(\tau)=f_{k}^{k+1}(\tau)$. Furthermore, proceeding analogously as with (3.8) we get

$$
K_{-}^{r}\left[e^{\mathrm{i} m \varphi} f(\tau)\right]=e^{\mathrm{i}(m-r) \varphi}\left(\cosh ^{-m+r} \tau \frac{d^{r}}{d(\sinh \tau)^{r}} \cosh ^{m} \tau\right) f(\tau) .
$$

Eqs. (2.12), (3.26), (3.4) and (3.7) taken together yield

$\tilde{\mathcal{Y}}_{k}^{m}(\tau, \varphi)=2^{k} k ! \sqrt{\frac{2 k+1}{2 \pi^{2}[-(m+k+1)] !(k-m) !}} e^{\mathrm{i} m \varphi} \cosh ^{-m} \tau \frac{d^{-(m+k+1)}}{d(\sinh \tau)^{-(m+k+1)}} \cosh ^{-2(k+1)} \tau$

The pseudospherical functions $\tilde{\mathcal{Y}}_{k}^{m}(\tau, \varphi)$ can be expressed by means of the functions $\mathcal{P}_{k}^{m}(\sinh \tau)$ defined by (3.11) in the form

$\tilde{\mathcal{Y}}_{k}^{m}(\tau, \varphi)=(-1)^{m+k+1} \sqrt{\frac{2 k+1}{2 \pi^{2}[-(m+k+1)] !(k-m) !}} e^{\mathrm{i} m \varphi} \mathcal{P}_{k}^{-m}(\sinh \tau)$.

Comparing (3.28) and (3.10) we obtain the following relationship between the pseudospherical functions $\mathcal{Y}_{k}^{m}$ and $\tilde{\mathcal{Y}}_{k}^{m}$ referring to the discrete series $D_{+}$and $D_{-}$, respectively:

$$
\left[\mathcal{Y}_{k}^{m}(\tau, \varphi)\right]^{*}=(-1)^{m-k-1} \tilde{\mathcal{Y}}_{k}^{-m}(\tau, \varphi) .
$$

For the sake of completeness we finally write down the Laplace-Beltrami operator in the biharmonic coordinates (2.16)

$$
\begin{aligned}
\Delta & =\left(\frac{\partial}{\partial x^{1}}\right)^{2}+\left(\frac{\partial}{\partial x^{2}}\right)^{2}-\left(\frac{\partial}{\partial x^{3}}\right)^{2}=\frac{1}{a} \frac{\partial^{2}}{\partial a^{2}} a-\frac{1}{a^{2}}\left(\frac{\partial^{2}}{\partial \tau^{2}}+\operatorname{tgh} \tau \frac{\partial}{\partial \tau}-\frac{1}{\cosh ^{2} \tau} \frac{\partial^{2}}{\partial \varphi^{2}}\right) \\
& =\frac{1}{a} \frac{\partial^{2}}{\partial a^{2}} a-\frac{1}{a^{2}} C_{2},
\end{aligned}
$$

where $C_{2}$ is the Casimir operator given by (2.18). It follows directly from (3.30) and (2.19a) that the functions defined by

$$
p_{k}^{m}(a)=a^{k} \mathcal{Y}_{k}^{m}(\tau, \varphi),
$$


fulfil

$$
\Delta p_{k}^{m}(a)=0 .
$$

The functions $p_{k}^{m}(a)$ are hyperbolic counterparts of the harmonic polynomials also called volume spherical functions.

\section{A new class of pseudospherical functions}

We now return to (2.21). Motivated by the a priori possible joining of the series $D_{+}$ and $D_{-}$we relax from the requirement $m \geq k+1$ for the series $D_{+}$and we study the case $m=k$ in (2.21). We then have

$$
\frac{d^{2} f_{k}^{k}(\tau)}{d \tau^{2}}+\operatorname{tgh} \tau \frac{d f_{k}^{k}(\tau)}{d \tau}-\left(k^{2} \operatorname{tgh}^{2} \tau+k\right) f_{k}^{k}(\tau)=0 .
$$

We point out that the same equation is obtained from (2.21) for $m=-k$. Hence we get $f_{k}^{-k}(\tau)=f_{k}^{k}(\tau)$. On introducing the new functions $g_{k}$ such that

$$
f_{k}^{k}(\tau)=g_{k}(\operatorname{tgh} \tau)
$$

we arrive at the equation

$$
g_{k}^{\prime \prime}(x)-\frac{x}{1-x^{2}} g_{k}^{\prime}(x)-\frac{k^{2} x^{2}+k}{\left(1-x^{2}\right)^{2}} g_{k}(x)=0 .
$$

The general solution to (4.3) is given by (see [19], eq. (2.76a))

$$
g_{k}(x)=\left(1-x^{2}\right)^{-\frac{k}{2}}\left[\alpha_{k}+\beta_{k} \int\left(1-x^{2}\right)^{k-\frac{1}{2}} d x\right],
$$

where $\alpha_{k}$ and $\beta_{k}$ are integration constants. From (4.4) we immediately get

$$
\begin{aligned}
& g_{0}(x)=\alpha_{0}+\beta_{0} \arcsin x, \\
& g_{1}(x)=\left(1-x^{2}\right)^{-\frac{1}{2}}\left\{\alpha_{1}+\frac{\beta_{1}}{2}\left[x\left(1-x^{2}\right)^{\frac{1}{2}}+\arcsin x\right]\right\} .
\end{aligned}
$$

The remaining functions $g_{k}(x), k \geq 2$, can be obtained from (4.4) and the following identity [17]:

$$
\begin{aligned}
& \int\left(1-x^{2}\right)^{k-\frac{1}{2}} d x=\frac{x\left(1-x^{2}\right)^{\frac{1}{2}}}{2 k}\left[\left(1-x^{2}\right)^{k-1}\right. \\
& \left.+\sum_{r=1}^{k-1} \frac{(2 k-1)(2 k-3) \cdots[2 k-(2 r-1)]}{2^{r}(k-1)(k-2) \cdots(k-r)}\left(1-x^{2}\right)^{k-1-r}\right]+\frac{(2 k-1) ! !}{2^{k} k !} \arcsin x,
\end{aligned}
$$

which can be written in a simpler form

$$
\begin{aligned}
& \int\left(1-x^{2}\right)^{k-\frac{1}{2}} d x=\frac{x\left(1-x^{2}\right)^{\frac{1}{2}}}{2 k}\left[\left(1-x^{2}\right)^{k-1}\right. \\
& \left.+\frac{(2 k-1) ! !}{2^{k}(k-1) !\left(1-x^{2}\right)} \sum_{r=1}^{k-1} \frac{2^{r}(r-1) !}{(2 r-1) ! !}\left(1-x^{2}\right)^{r}\right]+\frac{(2 k-1) ! !}{2^{k} k !} \arcsin x, \quad k \geq 2 .
\end{aligned}
$$


Now eqs. (2.20), (4.2), (4.4) and (4.6) taken together yield

$\mathcal{Y}_{0}^{0}(\tau, \varphi)=\alpha_{0}+\beta_{0} \arcsin \operatorname{tgh} \tau$

$\mathcal{Y}_{1}^{1}(\tau, \varphi)=\alpha_{1} e^{\mathrm{i} \varphi} \cosh \tau+\frac{\beta_{1}}{2} e^{\mathrm{i} \varphi}(\operatorname{tgh} \tau+\cosh \tau \arcsin \operatorname{tgh} \tau)$

$\mathcal{Y}_{k}^{k}(\tau)=\alpha_{k} e^{\mathrm{i} k \varphi} \cosh ^{k} \tau+\beta_{k} e^{\mathrm{i} k \varphi} \cosh ^{k} \tau\left\{\frac{\operatorname{tgh} \tau}{2 k \cosh \tau}\left[\cosh ^{-2 k+2} \tau\right.\right.$

$\left.\left.+\frac{(2 k-1) ! ! \cosh ^{2} \tau}{2^{k}(k-1) !} \sum_{r=1}^{k-1} \frac{2^{r}(r-1) !}{(2 r-1) ! !} \cosh ^{-2 r} \tau\right]+\frac{(2 k-1) ! !}{2^{k} k !} \arcsin \operatorname{tgh} \tau\right\}, \quad k \geq 2,(4.7 c)$

where the use can be made of the identity

$$
\arcsin \operatorname{tgh} \tau=2 \operatorname{arctg} e^{\tau}-\frac{\pi}{2} .
$$

As far as we are aware the formulas (4.7) on the pseudospherical functions are new. The functions $\mathcal{Y}_{k}^{k}(\tau, \varphi), k=0,1,2, \ldots$, and

$$
\tilde{\mathcal{Y}}_{k}^{-k}(\tau, \varphi)=e^{-\mathrm{i} k \varphi} f_{k}^{-k}(\tau)=e^{-\mathrm{i} k \varphi} f_{k}^{k}(\tau)
$$

are related to the series $D_{-}$and $D_{+}$, respectively. Namely, we have

$$
K_{-}^{2 k+1} \mathcal{Y}_{k}^{k} \sim \tilde{\mathcal{Y}}_{k}^{-(k+1)}, \quad K_{+}^{2 k+1} \tilde{\mathcal{Y}}_{k}^{-k} \sim \mathcal{Y}_{k}^{k+1} .
$$

Notice that by virtue of (4.10), in the case of $k=0$, the function $\mathcal{Y}_{0}^{0}$ really joins both series $D_{+}$and $D_{-}$via the ladder operators $K_{+}$and $K_{-}$. Finally, we remark that the functions $\mathcal{Y}_{k}^{k}(\tau, \varphi)$ are not square integrable on the one-sheet hyperboloid. It should also be noted that the function $\mathcal{Y}_{0}^{0}$ for $\beta_{0}=0$ is constant and refers to the trivial representation.

\section{Pseudospherical functions for continuous principal series}

\subsection{Normalization of pseudospherical functions}

In this section we study the pseudospherical functions for continuous principal series. In the case of such series we have neither highest nor lowest weight states and therefore the spherical functions cannot be found by solving any first-order differential equation as for the discrete series and application of the ladder operators. As a consequence, we have two kinds of pseudospherical functions referring to two linearly independent solutions of the second order equation (2.21). In view of (3.16) and the form of the parameter $k$ in the case of the continuous principal series such that $k=-\frac{1}{2}+\mathrm{i} \lambda$, these functions can be written as

$\hat{\mathcal{Y}}_{\lambda}^{m}(\tau, \varphi)=e^{\mathrm{i} m \varphi} \hat{c}_{m \lambda} \cosh ^{-\left(\frac{1}{2}+\mathrm{i} \lambda\right)} \tau_{2} F_{1}\left[\frac{1}{2}\left(m+\frac{1}{2}+\mathrm{i} \lambda\right), \frac{1}{2}\left(-m+\frac{1}{2}+\mathrm{i} \lambda\right) ; \frac{1}{2} ; \operatorname{tgh}^{2} \tau\right]$,

$\overline{\mathcal{Y}}_{\lambda}^{m}(\tau, \varphi)=e^{\mathrm{i} m \varphi} \bar{c}_{m \lambda} \cosh ^{-\left(\frac{1}{2}+\mathrm{i} \lambda\right)} \tau \operatorname{tgh} \tau_{2} F_{1}\left[\frac{1}{2}\left(m+\frac{3}{2}+\mathrm{i} \lambda\right), \frac{1}{2}\left(-m+\frac{3}{2}+\mathrm{i} \lambda\right) ; \frac{3}{2} ; \operatorname{tgh}^{2} \tau\right]$,

where $\hat{c}_{m \lambda}$ and $\bar{c}_{m \lambda}$ are normalization constants. Following the algoritm described in [4] we now normalize the function $\hat{\mathcal{Y}}_{\lambda}^{m}(\tau, \varphi)$. Firstly, using the formula on the analytic 
continuation [20] such that

$$
\begin{aligned}
& { }_{2} F_{1}(\alpha, \beta ; \gamma ; z)=A_{12} F_{1}(\alpha, \beta ; \alpha+\beta-\gamma+1 ; 1-z) \\
& \quad+A_{2}(1-z)^{\gamma-\alpha-\beta}{ }_{2} F_{1}(\gamma-\alpha, \gamma-\beta ; \gamma-\alpha-\beta+1 ; 1-z), \quad|\arg (1-z)|<\pi,
\end{aligned}
$$

where

$$
A_{1}=\frac{\Gamma(\gamma) \Gamma(\gamma-\alpha-\beta)}{\Gamma(\gamma-\alpha) \Gamma(\gamma-\beta)}, \quad A_{2}=\frac{\Gamma(\gamma) \Gamma(\alpha+\beta-\gamma)}{\Gamma(\alpha) \Gamma(\beta)}
$$

we get

$$
\begin{aligned}
{ }_{2} F_{1}\left[\frac{1}{2}\left(m+\frac{1}{2}+\mathrm{i} \lambda\right), \frac{1}{2}\left(-m+\frac{1}{2}+\mathrm{i} \lambda\right) ; \frac{1}{2} ; \operatorname{tgh}^{2} \tau\right] \\
=\hat{A}_{12} F_{1}\left[\frac{1}{2}\left(m+\frac{1}{2}+\mathrm{i} \lambda\right), \frac{1}{2}\left(-m+\frac{1}{2}+\mathrm{i} \lambda\right) ; 1+\mathrm{i} \lambda ; \frac{1}{\cosh ^{2} \tau}\right] \\
\quad+\hat{A}_{2} \cosh ^{2 \mathrm{i} \lambda} \tau_{2} F_{1}\left[\frac{1}{2}\left(-m+\frac{1}{2}-\mathrm{i} \lambda\right), \frac{1}{2}\left(m+\frac{1}{2}-\mathrm{i} \lambda\right) ; 1-\mathrm{i} \lambda ; \frac{1}{\cosh ^{2} \tau}\right],
\end{aligned}
$$

where

$$
\begin{aligned}
& \hat{A}_{1}=\frac{\sqrt{\pi} \Gamma(-\mathrm{i} \lambda)}{\Gamma\left[\frac{1}{2}\left(-m+\frac{1}{2}-\mathrm{i} \lambda\right)\right] \Gamma\left[\frac{1}{2}\left(m+\frac{1}{2}-\mathrm{i} \lambda\right)\right]}, \\
& \hat{A}_{2}=\hat{A}_{1}^{*}=\frac{\sqrt{\pi} \Gamma(\mathrm{i} \lambda)}{\Gamma\left[\frac{1}{2}\left(m+\frac{1}{2}+\mathrm{i} \lambda\right)\right] \Gamma\left[\frac{1}{2}\left(-m+\frac{1}{2}+\mathrm{i} \lambda\right)\right]} .
\end{aligned}
$$

Hence, taking into account the relations

$$
\lim _{\tau \rightarrow \pm \infty} \cosh \tau=\frac{e^{ \pm \tau}}{2}, \quad{ }_{2} F_{1}(\alpha, \beta ; \gamma ; 0)=1
$$

we obtain the asymptotic form of $\hat{\mathcal{Y}}_{\lambda}^{m}(\tau, \varphi)$. Namely, we have

$$
\lim _{\tau \rightarrow \infty} \hat{\mathcal{Y}}_{\lambda}^{m}(\tau, \varphi)=e^{\mathrm{i} m \varphi} \hat{c}_{m \lambda} \cosh ^{-\frac{1}{2}} \tau\left(B e^{-\mathrm{i} \lambda \tau}+B^{*} e^{\mathrm{i} \lambda \tau}\right),
$$

where $|B|=\left|\hat{A}_{1}\right|$. On demanding that asymptotic functions are normalized as (see $(3.5))$

$$
\int_{-\infty}^{\infty} \cosh \tau d \tau \int_{0}^{2 \pi} d \varphi\left[\hat{\mathcal{Y}}_{\lambda}^{m}(\tau, \varphi)\right]^{*} \hat{\mathcal{Y}}_{\lambda^{\prime}}^{m^{\prime}}(\tau, \varphi)=\delta_{m m^{\prime}} \delta\left(\lambda-\lambda^{\prime}\right)
$$

we arrive at the following formula on the normalization constant

$$
\left|\hat{c}_{m \lambda}\right|^{2}=\frac{1}{8 \pi^{2}\left|\hat{A}_{1}\right|^{2}}=\frac{1}{8 \pi^{3}} \frac{\left|\Gamma\left[\frac{1}{2}\left(m+\frac{1}{2}+\mathrm{i} \lambda\right)\right] \Gamma\left[\frac{1}{2}\left(-m+\frac{1}{2}+\mathrm{i} \lambda\right)\right]\right|^{2}}{|\Gamma(\mathrm{i} \lambda)|^{2}} .
$$

We now normalize the function $\overline{\mathcal{Y}}_{\lambda}^{m}(\tau, \varphi)$. Using (5.3) and (5.4) we find $\operatorname{tgh} \tau_{2} F_{1}\left[\frac{1}{2}\left(m+\frac{3}{2}+\mathrm{i} \lambda\right), \frac{1}{2}\left(-m+\frac{3}{2}+\mathrm{i} \lambda\right) ; \frac{3}{2} ; \operatorname{tgh}^{2} \tau\right]$

$$
\begin{aligned}
= & \operatorname{tgh} \tau\left\{\bar{A}_{12} F_{1}\left[\frac{1}{2}\left(m+\frac{3}{2}+\mathrm{i} \lambda\right), \frac{1}{2}\left(-m+\frac{3}{2}+\mathrm{i} \lambda\right) ; 1+\mathrm{i} \lambda ; \frac{1}{\cosh ^{2} \tau}\right]\right. \\
& \left.+\bar{A}_{2} \cosh ^{2 \mathrm{i} \lambda} \tau_{2} F_{1}\left[\frac{1}{2}\left(-m+\frac{3}{2}-\mathrm{i} \lambda\right), \frac{1}{2}\left(m+\frac{3}{2}-\mathrm{i} \lambda\right) ; 1+\mathrm{i} \lambda ; \frac{1}{\cosh ^{2} \tau}\right]\right\},
\end{aligned}
$$


where

$$
\begin{aligned}
& \bar{A}_{1}=\frac{\sqrt{\pi}}{2} \frac{\Gamma(-\mathrm{i} \lambda)}{\Gamma\left[\frac{1}{2}\left(-m+\frac{3}{2}-\mathrm{i} \lambda\right)\right] \Gamma\left[\frac{1}{2}\left(m+\frac{3}{2}-\mathrm{i} \lambda\right)\right]} \\
& \bar{A}_{2}=\left(\bar{A}_{1}\right)^{*}=\frac{\sqrt{\pi}}{2} \frac{\Gamma(\mathrm{i} \lambda)}{\Gamma\left[\frac{1}{2}\left(m+\frac{3}{2}+\mathrm{i} \lambda\right)\right] \Gamma\left[\frac{1}{2}\left(-m+\frac{3}{2}+\mathrm{i} \lambda\right)\right]} .
\end{aligned}
$$

Proceeding analogously as in the case of the normalization of the function $\hat{\mathcal{Y}}_{\lambda}^{m}(\tau, \varphi)$, and making use of of the $\operatorname{limit} \lim _{\tau \rightarrow \pm \infty} \operatorname{tgh} \tau= \pm 1$, we obtain

$$
\left|\bar{c}_{m \lambda}\right|^{2}=\frac{1}{8 \pi^{2}\left|\bar{A}_{1}\right|^{2}}=\frac{1}{2 \pi^{3}} \frac{\left|\Gamma\left[\frac{1}{2}\left(m+\frac{3}{2}+\mathrm{i} \lambda\right)\right] \Gamma\left[\frac{1}{2}\left(-m+\frac{3}{2}+\mathrm{i} \lambda\right)\right]\right|^{2}}{|\Gamma(\mathrm{i} \lambda)|^{2}} .
$$

Taking into account (5.1), (5.2), (5.10), and (5.13) we can finally write down the desired normalized pseudospherical functions

$$
\begin{aligned}
& \hat{\mathcal{Y}}_{\lambda}^{m}(\tau, \varphi)=\hat{c}_{m} \frac{e^{\mathrm{i} m \varphi}}{2 \sqrt{2} \pi^{\frac{3}{2}}} \frac{\left|\Gamma\left[\frac{1}{2}\left(m+\frac{1}{2}+\mathrm{i} \lambda\right)\right] \Gamma\left[\frac{1}{2}\left(-m+\frac{1}{2}+\mathrm{i} \lambda\right)\right]\right|}{|\Gamma(\mathrm{i} \lambda)|} \cosh ^{-\left(\frac{1}{2}+\mathrm{i} \lambda\right)} \tau \\
& \times{ }_{2} F_{1}\left[\frac{1}{2}\left(m+\frac{1}{2}+\mathrm{i} \lambda\right), \frac{1}{2}\left(-m+\frac{1}{2}+\mathrm{i} \lambda\right) ; \frac{1}{2} ; \operatorname{tgh}^{2} \tau\right], \\
& \overline{\mathcal{Y}}_{\lambda}^{m}(\tau, \varphi)=\bar{c}_{m} \frac{e^{\mathrm{i} m \varphi}}{\sqrt{2} \pi^{\frac{3}{2}}} \frac{\left|\Gamma\left[\frac{1}{2}\left(m+\frac{3}{2}+\mathrm{i} \lambda\right)\right] \Gamma\left[\frac{1}{2}\left(-m+\frac{3}{2}+\mathrm{i} \lambda\right)\right]\right|}{|\Gamma(\mathrm{i} \lambda)|} \cosh ^{-\left(\frac{1}{2}+\mathrm{i} \lambda\right)} \tau \operatorname{tgh} \tau \\
& \times{ }_{2} F_{1}\left[\frac{1}{2}\left(m+\frac{3}{2}+\mathrm{i} \lambda\right), \frac{1}{2}\left(-m+\frac{3}{2}+\mathrm{i} \lambda\right) ; \frac{3}{2} ; \operatorname{tgh}^{2} \tau\right] .
\end{aligned}
$$

where $\hat{c}_{m}$ and $\bar{c}_{m}$ are phases that will be fixed in the next section. We point out that obtained normalization of pseudospherical functions provides the most probably new nontrivial identities for the hypergeometric functions.

\subsection{The Fock space structure of the continuous principal series representation}

Although in the case of the continuous series representation there is neither lowest nor highest weight states, nevertheless there is still possibility, in view of (2.6), of generation of states with arbitrary $m$ from some fixed one by means of the ladder operators $K_{ \pm}$. This possibility is the subject of this section. Now, it seems that the most natural candidates for such a distinguished "vacuum vector" in the case with integer $m$, is the state with $m=0$. Taking into account (2.6) we find for $k=-\frac{1}{2}+\mathrm{i} \lambda$

$$
\begin{aligned}
& K_{+}|\lambda, m\rangle=\sqrt{\left(m+\frac{1}{2}\right)^{2}+\lambda^{2}}|\lambda, m+1\rangle, \\
& K_{-}|\lambda, m\rangle=\sqrt{\left(m-\frac{1}{2}\right)^{2}+\lambda^{2}}|\lambda, m-1\rangle,
\end{aligned}
$$

where $|\lambda, m\rangle \equiv\left|-\frac{1}{2}+\mathrm{i} \lambda, m\right\rangle$. An easy calculation based on (5.16a) shows that

$$
|\lambda, m\rangle=\frac{1}{\prod_{r=1}^{m} \sqrt{\left(\frac{2 r-1}{2}\right)^{2}+\lambda^{2}}} K_{+}^{m}|\lambda, 0\rangle, \quad m=1,2, \ldots
$$

We remark that the fraction from (5.17) can be expressed by the gamma functions. Namely, using the identity [21]

$$
\frac{\Gamma\left(m+\frac{1}{2}-\mathrm{i} \lambda\right)}{\Gamma\left(-m+\frac{1}{2}-\mathrm{i} \lambda\right)}=(-1)^{m} \prod_{r=1}^{m}\left[\left(\frac{2 r-1}{2}\right)^{2}+\lambda^{2}\right]
$$


and [17]

$$
\Gamma(z)=\frac{2^{z-1}}{\sqrt{\pi}} \Gamma\left(\frac{1}{2} z\right) \Gamma\left[\frac{1}{2}(z+1)\right]
$$

one can show that

$$
\begin{aligned}
\frac{1}{\prod_{r=1}^{m} \sqrt{\left(\frac{2 r-1}{2}\right)^{2}+\lambda^{2}}} & =2^{-m} \frac{\left|\Gamma\left[\frac{1}{2}\left(-m+\frac{1}{2}+\mathrm{i} \lambda\right)\right]\right|}{\left|\Gamma\left[\frac{1}{2}\left(m+\frac{1}{2}+\mathrm{i} \lambda\right)\right]\right|}, \\
& =2^{-m} \frac{\left|\Gamma\left[\frac{1}{2}\left(-m+\frac{3}{2}+\mathrm{i} \lambda\right)\right]\right|}{\left|\Gamma\left[\frac{1}{2}\left(m+\frac{3}{2}+\mathrm{i} \lambda\right)\right]\right|} .
\end{aligned}
$$

We remark that the right-hand side of (5.18) is usually designated by $Z_{\lambda}^{m}$. Now, we have in our disposal two possibilities to choose the function representing vector $|\lambda, 0\rangle$, namely $\hat{\mathcal{Y}}_{\lambda}^{0}$, and $\overline{\mathcal{Y}}_{\lambda}^{0}$ (see (5.1) and (5.2)), and therefore two different sequences of pseudospherical functions generated via (5.17). Let us designate these sequences by $\mathcal{Y}_{1 \lambda}^{m}$ and $\mathcal{Y}_{2 \lambda}^{m}$, respectively. In order to identify elements of $\mathcal{Y}_{1 \lambda}^{m}$ and $\mathcal{Y}_{2 \lambda}^{m}$ we first point out that the functions $\hat{\mathcal{Y}}_{\lambda}^{m}$ are even and the functions $\overline{\mathcal{Y}}_{\lambda}^{m}$ are odd functions of $\tau$ for arbitrary $m$. Furthermore, it follows immediately from the form $(2.17 \mathrm{a})$ of the operator $K_{+}$that it maps an even function to odd one and vice versa. Therefore, the sequence $\mathcal{Y}_{1_{\lambda}}^{m}$ is of the form

$$
\hat{\mathcal{Y}}_{\lambda}^{0}, \overline{\mathcal{Y}}_{\lambda}^{1}, \hat{\mathcal{Y}}_{\lambda}^{2}, \ldots, \hat{\mathcal{Y}}_{\lambda}^{2 n}, \overline{\mathcal{Y}}_{\lambda}^{2 n+1}, \ldots
$$

that is

$$
\mathcal{Y}_{1 \lambda}^{m}= \begin{cases}\hat{\mathcal{Y}}_{\lambda}^{m}, & \text { for } m=2 n, \\ \overline{\mathcal{Y}}_{\lambda}^{m}, & \text { for } m=2 n+1, \quad n=0,1, \ldots\end{cases}
$$

Analogously, the sequence $\mathcal{Y}_{2 \lambda}^{m}$ is given by

$$
\overline{\mathcal{Y}}_{\lambda}^{0}, \hat{\mathcal{Y}}_{\lambda}^{1}, \overline{\mathcal{Y}}_{\lambda}^{2}, \ldots, \overline{\mathcal{Y}}_{\lambda}^{2 n}, \hat{\mathcal{Y}}_{\lambda}^{2 n+1}, \ldots
$$

i.e.

$$
\mathcal{Y}_{2 \lambda}^{m}= \begin{cases}\overline{\mathcal{Y}}_{\lambda}^{m}, & \text { for } m=2 n, \\ \hat{\mathcal{Y}}_{\lambda}^{m}, & \text { for } m=2 n+1, \quad n=0,1, \ldots\end{cases}
$$

Surprisingly, this structure of the principal series was not, to our best knowledge, discussed in the literature. We stress that in the case of the discrete series all functions, even and odd ones, are elements of the only one sequence of pseudospherical functions starting with the unique highest or lowest weight state (for the series $D_{+}$see (3.16)). We now derive the counterpart of the formula (3.15) in the case of the continuous series. We begin with the case of the functions $\mathcal{Y}_{1 \lambda}^{m}$. To this end we first write down the function $\hat{\mathcal{Y}}_{\lambda}^{0}(\tau, \varphi)$ following directly from $(5.14)$

$\hat{\mathcal{Y}}_{\lambda}^{0}(\tau, \varphi)=\frac{1}{2 \sqrt{2} \pi^{\frac{3}{2}}} \frac{\left|\Gamma\left[\frac{1}{2}\left(\frac{1}{2}+\mathrm{i} \lambda\right)\right]\right|^{2}}{|\Gamma(\mathrm{i} \lambda)|} \cosh ^{-\left(\frac{1}{2}+\mathrm{i} \lambda\right)} \tau_{2} F_{1}\left[\frac{1}{2}\left(\frac{1}{2}+\mathrm{i} \lambda\right), \frac{1}{2}\left(\frac{1}{2}+\mathrm{i} \lambda\right) ; \frac{1}{2} ; \operatorname{tgh}^{2} \tau\right]$,

where we choose, without loose of generality, the phase so $\hat{c}_{0}=1$. Using the identity [21]

$$
{ }_{2} F_{1}(\alpha, \beta ; \gamma ; z)=(1-z)^{-\alpha}{ }_{2} F_{1}\left(\alpha, \gamma-\beta ; \gamma ; \frac{z}{z-1}\right)
$$


we obtain the following equivalent form of the function $\hat{\mathcal{Y}}_{\lambda}^{0}$ such that

$\hat{\mathcal{Y}}_{\lambda}^{0}(\tau, \varphi)=\frac{1}{2 \sqrt{2} \pi^{\frac{3}{2}}} \frac{\left|\Gamma\left[\frac{1}{2}\left(\frac{1}{2}+\mathrm{i} \lambda\right)\right]\right|^{2}}{|\Gamma(\mathrm{i} \lambda)|}{ }_{2} F_{1}\left[\frac{1}{2}\left(\frac{1}{2}+\mathrm{i} \lambda\right), \frac{1}{2}\left(\frac{1}{2}-\mathrm{i} \lambda\right) ; \frac{1}{2} ;-\sinh ^{2} \tau\right]$.

It follows immediately from (3.8) and (5.17) that the pseudospherical functions $\mathcal{Y}_{1 \lambda}^{m}$ are given by

$$
\begin{aligned}
\mathcal{Y}_{1 \lambda}^{m}(\tau, \varphi)= & (-1)^{m} \frac{1}{2 \sqrt{2} \pi^{\frac{3}{2}}} \frac{\left|\Gamma\left[\frac{1}{2}\left(\frac{1}{2}+\mathrm{i} \lambda\right)\right]\right|^{2}}{|\Gamma(\mathrm{i} \lambda)|} \frac{1}{\prod_{r=1}^{m} \sqrt{\left(\frac{2 r-1}{2}\right)^{2}+\lambda^{2}}} e^{\mathrm{i} m \varphi} \cosh ^{m} \tau \\
& \times \frac{d^{m}}{d(\sinh \tau)^{m}}{ }_{2} F_{1}\left[\frac{1}{2}\left(\frac{1}{2}+\mathrm{i} \lambda\right), \frac{1}{2}\left(\frac{1}{2}-\mathrm{i} \lambda\right) ; \frac{1}{2} ;-\sinh ^{2} \tau\right] .
\end{aligned}
$$

Notice that the relation (5.28) can be written as (compare (3.10))

$\mathcal{Y}_{1 \lambda}^{m}(\tau, \varphi)=\frac{1}{2 \sqrt{2} \pi^{\frac{3}{2}}} \frac{1}{\prod_{r=1}^{m} \sqrt{\left(\frac{2 r-1}{2}\right)^{2}+\lambda^{2}}} e^{\mathrm{i} m \varphi} \mathcal{P}_{1_{\lambda}}^{m}(\sinh \tau)$,

where $\mathcal{P}_{1 \lambda}^{m}(x)$ are the plausible pseudospherical counterparts of the Legendre functions defined as

$\mathcal{P}_{1 \lambda}^{m}(x)=(-1)^{m} \frac{\left|\Gamma\left[\frac{1}{2}\left(\frac{1}{2}+\mathrm{i} \lambda\right)\right]\right|^{2}}{|\Gamma(\mathrm{i} \lambda)|}\left(1+x^{2}\right)^{\frac{m}{2}} \frac{d^{m}}{d x^{m}}{ }_{2} F_{1}\left[\frac{1}{2}\left(\frac{1}{2}+\mathrm{i} \lambda\right), \frac{1}{2}\left(\frac{1}{2}-\mathrm{i} \lambda\right) ; \frac{1}{2} ;-x^{2}\right]$.

Now, taking into account (3.12) we get

$$
\begin{aligned}
& \frac{d^{m}}{d x^{m}}{ }_{2} F_{1}\left[\frac{1}{2}\left(\frac{1}{2}+\mathrm{i} \lambda\right), \frac{1}{2}\left(\frac{1}{2}-\mathrm{i} \lambda\right) ; \frac{1}{2} ;-x^{2}\right] \\
& \quad=\left.m ! \sum_{r=0}^{\left[\frac{m}{2}\right]} \frac{(2 x)^{m-2 r}}{r !(m-2 r) !}\left(\frac{d^{m-r}}{d x^{m-r}}{ }_{2} F_{1}\left[\frac{1}{2}\left(\frac{1}{2}+\mathrm{i} \lambda\right), \frac{1}{2}\left(\frac{1}{2}-\mathrm{i} \lambda\right) ; \frac{1}{2} ;-x\right]\right)\right|_{x=x^{2}} .
\end{aligned}
$$

Hence, using the identity [20]

$$
\frac{d^{n}}{d z^{n}}{ }_{2} F_{1}(\alpha, \beta ; \gamma ; z)=\frac{\Gamma(\alpha+n) \Gamma(\beta+n) \Gamma(\gamma)}{\Gamma(\alpha) \Gamma(\beta) \Gamma(\gamma+n)}{ }_{2} F_{1}(\alpha+n, \beta+n ; \gamma+n ; z)
$$

we find

$$
\begin{aligned}
\mathcal{P}_{1 \lambda}^{m}(x)= & \frac{\sqrt{\pi} m !}{|\Gamma(\mathrm{i} \lambda)|}\left(1+x^{2}\right)^{\frac{m}{2}} \sum_{r=0}^{\left[\frac{m}{2}\right]}(-1)^{r} \frac{(2 x)^{m-2 r}}{r !(m-2 r) !} \frac{\left|\Gamma\left[\frac{1}{2}\left(\frac{1}{2}-\mathrm{i} \lambda\right)+m-r\right]\right|^{2}}{\Gamma\left(\frac{1}{2}+m-r\right)} \\
& \times{ }_{2} F_{1}\left[\frac{1}{2}\left(\frac{1}{2}+\mathrm{i} \lambda\right)+m-r, \frac{1}{2}\left(\frac{1}{2}-\mathrm{i} \lambda\right)+m-r ; \frac{1}{2}+m-r ;-x^{2}\right] .
\end{aligned}
$$

Substituting $x=\sinh \tau$ and making use of (5.26) we obtain

$$
\begin{aligned}
\mathcal{P}_{1 \lambda}^{m}(\sinh \tau)= & \frac{\sqrt{\pi} m !}{|\Gamma(\mathrm{i} \lambda)|} \cosh ^{-\left(\frac{1}{2}+\mathrm{i} \lambda\right)} \tau \sum_{r=0}^{\left[\frac{m}{2}\right]}(-1)^{r} \frac{(2 \operatorname{tgh} \tau)^{m-2 r}}{r !(m-2 r) !} \frac{\left|\Gamma\left[\frac{1}{2}\left(\frac{1}{2}-\mathrm{i} \lambda\right)+m-r\right]\right|^{2}}{\Gamma\left(\frac{1}{2}+m-r\right)} \\
& \times{ }_{2} F_{1}\left[\frac{1}{2}\left(\frac{1}{2}+\mathrm{i} \lambda\right)+m-r, \frac{1}{2}\left(\frac{1}{2}+\mathrm{i} \lambda\right) ; \frac{1}{2}+m-r ; \operatorname{tgh}^{2} \tau\right] .
\end{aligned}
$$

Therefore, the pseudospherical functions $\mathcal{Y}_{1_{\lambda}}^{m}(\tau, \varphi)$ can be written in the form

$$
\begin{gathered}
\mathcal{Y}_{1 \lambda}^{m}(\tau, \varphi)=\frac{m !}{2 \sqrt{2} \pi|\Gamma(\mathrm{i} \lambda)|} \frac{1}{\prod_{r=1}^{m} \sqrt{\left(\frac{2 r-1}{2}\right)^{2}+\lambda^{2}}} e^{\mathrm{i} m \varphi} \cosh ^{-\left(\frac{1}{2}+\mathrm{i} \lambda\right)} \tau \sum_{r=0}^{\left[\frac{m}{2}\right]}(-1)^{r} \frac{(2 \operatorname{tgh} \tau)^{m-2 r}}{r !(m-2 r) !} \\
\times \frac{\left|\Gamma\left[\frac{1}{2}\left(\frac{1}{2}-\mathrm{i} \lambda\right)+m-r\right]\right|^{2}}{\Gamma\left(\frac{1}{2}+m-r\right)}{ }_{2} F_{1}\left[\frac{1}{2}\left(\frac{1}{2}+\mathrm{i} \lambda\right)+m-r, \frac{1}{2}\left(\frac{1}{2}+\mathrm{i} \lambda\right) ; \frac{1}{2}+m-r ; \operatorname{tgh}^{2} \tau\right],(5.35)
\end{gathered}
$$


Pseudospherical functions on a hyperboloid of one sheet

where $m=1,2, \ldots$

We now discuss the pseudospherical functions $\mathcal{Y}_{2 \lambda}^{m}(\tau, \varphi)$. From (5.15) we immediately find that the odd "vacuum vector" $\overline{\mathcal{Y}}_{\lambda}^{0}(\tau, \varphi)$ is

$\overline{\mathcal{Y}}_{\lambda}^{0}(\tau, \varphi)=\frac{1}{\sqrt{2} \pi^{\frac{3}{2}}} \frac{\left|\Gamma\left[\frac{1}{2}\left(\frac{3}{2}+\mathrm{i} \lambda\right)\right]\right|^{2}}{|\Gamma(\mathrm{i} \lambda)|} \cosh ^{-\left(\frac{1}{2}+\mathrm{i} \lambda\right)} \tau \operatorname{tgh} \tau_{2} F_{1}\left[\frac{1}{2}\left(\frac{3}{2}+\mathrm{i} \lambda\right), \frac{1}{2}\left(\frac{3}{2}+\mathrm{i} \lambda\right) ; \frac{3}{2} ; \operatorname{tgh}^{2} \tau\right]$

where we set $\bar{c}=1$. Using (5.26) we get

$\overline{\mathcal{Y}}_{\lambda}^{0}(\tau, \varphi)=\frac{1}{\sqrt{2} \pi^{\frac{3}{2}}} \frac{\left|\Gamma\left[\frac{1}{2}\left(\frac{3}{2}+\mathrm{i} \lambda\right)\right]\right|^{2}}{|\Gamma(\mathrm{i} \lambda)|} \sinh \tau_{2} F_{1}\left[\frac{1}{2}\left(\frac{3}{2}+\mathrm{i} \lambda\right), \frac{1}{2}\left(\frac{3}{2}-\mathrm{i} \lambda\right) ; \frac{3}{2} ;-\sinh ^{2} \tau\right]$.

Taking into account (5.17) we arrive at the following formula on the pseudospherical functions $\mathcal{Y}_{2 \lambda}^{m}(\tau, \varphi)$ :

$$
\begin{aligned}
\mathcal{Y}_{2 \lambda}^{m}(\tau, \varphi)= & (-1)^{m} \frac{1}{\sqrt{2} \pi^{\frac{3}{2}}} \frac{\left|\Gamma\left[\frac{1}{2}\left(\frac{3}{2}+\mathrm{i} \lambda\right)\right]\right|^{2}}{|\Gamma(\mathrm{i} \lambda)|} \frac{1}{\prod_{r=1}^{m} \sqrt{\left(\frac{2 r-1}{2}\right)^{2}+\lambda^{2}}} e^{\mathrm{i} m \varphi} \cosh ^{m} \tau \\
& \times \frac{d^{m}}{d(\sinh \tau)^{m}} \sinh \tau_{2} F_{1}\left[\frac{1}{2}\left(\frac{3}{2}+\mathrm{i} \lambda\right), \frac{1}{2}\left(\frac{3}{2}-\mathrm{i} \lambda\right) ; \frac{3}{2} ;-\sinh ^{2} \tau\right] .
\end{aligned}
$$

Introducing the counterparts of the Legendre functions $\mathcal{P}_{2 \lambda}^{m}(x)$ we can write (5.38) in the form

$\mathcal{Y}_{2 \lambda}^{m}(\tau, \varphi)=\frac{1}{\sqrt{2} \pi^{\frac{3}{2}}} \frac{1}{\prod_{r=1}^{m} \sqrt{\left(\frac{2 r-1}{2}\right)^{2}+\lambda^{2}}} e^{\mathrm{i} m \varphi} \mathcal{P}_{2 \lambda}^{m}(\sinh \tau)$,

where

$\mathcal{P}_{2 \lambda}^{m}(x)=(-1)^{m} \frac{\left|\Gamma\left[\frac{1}{2}\left(\frac{3}{2}+\mathrm{i} \lambda\right)\right]\right|^{2}}{|\Gamma(\mathrm{i} \lambda)|}\left(1+x^{2}\right)^{\frac{m}{2}} \frac{d^{m}}{d x^{m}} x_{2} F_{1}\left[\frac{1}{2}\left(\frac{3}{2}+\mathrm{i} \lambda\right), \frac{1}{2}\left(\frac{3}{2}-\mathrm{i} \lambda\right) ; \frac{3}{2} ;-x^{2}\right]$.

Eqs. (5.40), (3.12) and (5.32) taken together yield

$$
\begin{aligned}
\mathcal{P}_{2 \lambda}{ }_{\lambda}^{m}(x)= & \frac{\sqrt{\pi}(m+1) !}{4|\Gamma(\mathrm{i} \lambda)|}\left(1+x^{2}\right)^{\frac{m}{2}} \sum_{r=0}^{\left[\frac{m+1}{2}\right]}(-1)^{r} \frac{(2 x)^{m+1-2 r}}{r !(m+1-2 r) !} \frac{\left|\Gamma\left[\frac{1}{2}\left(\frac{3}{2}+\mathrm{i} \lambda\right)+m-r\right]\right|^{2}}{\Gamma\left(\frac{3}{2}+m-r\right)} \\
& \times{ }_{2} F_{1}\left[\frac{1}{2}\left(\frac{3}{2}+\mathrm{i} \lambda\right)+m-r, \frac{1}{2}\left(\frac{3}{2}-\mathrm{i} \lambda\right)+m-r ; \frac{3}{2}+m-r ;-x^{2}\right] .
\end{aligned}
$$

Hence, using (5.26) we get

$$
\begin{aligned}
\mathcal{P}_{2 \lambda}^{m}(\sinh \tau)= & \frac{\sqrt{\pi}(m+1) !}{4|\Gamma(\mathrm{i} \lambda)|} \cosh ^{-\left(\frac{1}{2}+\mathrm{i} \lambda\right)} \tau \sum_{r=0}^{\left[\frac{m+1}{2}\right]}(-1)^{r} \frac{(2 \operatorname{tgh} \tau)^{m+1-2 r}}{r !(m+1-2 r) !} \frac{\left|\Gamma\left[\frac{1}{2}\left(\frac{3}{2}+\mathrm{i} \lambda\right)+m-r\right]\right|^{2}}{\Gamma\left(\frac{3}{2}+m-r\right)} \\
& \times{ }_{2} F_{1}\left[\frac{1}{2}\left(\frac{3}{2}+\mathrm{i} \lambda\right)+m-r, \frac{1}{2}\left(\frac{3}{2}+\mathrm{i} \lambda\right) ; \frac{3}{2}+m-r ; \operatorname{tgh}^{2} \tau\right] .
\end{aligned}
$$

An immediate consequence of (5.39) and (5.42) is the following formula on the pseudospherical functions $\mathcal{Y}_{2 \lambda}^{m}(\tau, \varphi)$ :

$$
\begin{gathered}
\mathcal{Y}_{2 \lambda}^{m}(\tau, \varphi)=\frac{(m+1) !}{4 \sqrt{2} \pi|\Gamma(\mathrm{i} \lambda)|} \frac{1}{\prod_{r=1}^{m} \sqrt{\left(\frac{2 r-1}{2}\right)^{2}+\lambda^{2}}} e^{\mathrm{i} m \varphi} \cosh ^{-\left(\frac{1}{2}+\mathrm{i} \lambda\right)} \tau \sum_{r=0}^{\left[\frac{m+1}{2}\right]}(-1)^{r} \frac{(2 \operatorname{tgh} \tau)^{m+1-2 r}}{r !(m+1-2 r) !} \\
\times \frac{\left|\Gamma\left[\frac{1}{2}\left(\frac{3}{2}+\mathrm{i} \lambda\right)+m-r\right]\right|^{2}}{\Gamma\left(\frac{3}{2}+m-r\right)}{ }_{2} F_{1}\left[\frac{1}{2}\left(\frac{3}{2}+\mathrm{i} \lambda\right)+m-r, \frac{1}{2}\left(\frac{3}{2}+\mathrm{i} \lambda\right) ; \frac{3}{2}+m-r ; \operatorname{tgh}^{2} \tau\right], \quad(5.43)
\end{gathered}
$$


where $m=1,2, \ldots$. An advantage of the relations (5.35) and (5.43) is that they enable to fix with the help of the Gauss relations between contiguous functions [21] the phases of the even and odd pseudospherical functions (5.14) and (5.15). Namely, we find

$$
\hat{c}_{m}= \begin{cases}(-1)^{\frac{m}{2}}, & \text { for } m=2 n, \\ (-1)^{\frac{m+1}{2}}, & \text { for } m=2 n+1, \quad n=0,1, \ldots\end{cases}
$$

and

$$
\bar{c}_{m}= \begin{cases}(-1)^{\frac{m}{2}}, & \text { for } m=2 n, \\ (-1)^{\frac{m-1}{2}}, & \text { for } m=2 n+1, \quad n=0,1, \ldots\end{cases}
$$

We stress that the phases cannot be fixed without knowledge of the infinite sequence of pseudospherical functions generated by means of the ladder operators.

We finally point out that an immediate consequence of the relation

$$
|\lambda,-m\rangle=\frac{1}{\prod_{r=1}^{m} \sqrt{\left(\frac{2 r-1}{2}\right)^{2}+\lambda^{2}}} K_{-}^{m}|\lambda, 0\rangle, \quad m=1,2, \ldots
$$

following directly from (5.16b) is the formula on $\mathcal{Y}_{1_{\lambda}}^{-m}$ and $\mathcal{Y}_{2_{\lambda}}^{-m}$ such that

$$
\mathcal{Y}_{1,2 \lambda}^{-m}(\tau, \varphi)=(-1)^{m}\left[\mathcal{Y}_{1,2 \lambda}^{m}(\tau, \varphi)\right]^{*}, \quad m=1,2, \ldots
$$

\subsection{Pseudospherical functions with half-integer $\mathrm{m}$}

In this section we study the pseudospherical functions $\mathcal{Y}_{1 \lambda}^{m}$ and $\mathcal{Y}_{2 \lambda}^{m}$ in the case of the continuous principal series and half-integer $m$. As far as we are aware the pseudospherical functions with half-integer $m$ were not discussed in the literature. We now return to (5.14). Taking into account the identity [20]

$$
{ }_{2} F_{1}\left(\alpha+\frac{1}{2}, \alpha ; \frac{1}{2} ; z\right)=\frac{1}{2}(1+\sqrt{z})^{-2 \alpha}+\frac{1}{2}(1-\sqrt{z})^{-2 \alpha}
$$

as well as the following properties of the gamma function [17]:

$$
\begin{aligned}
\left|\Gamma\left(\frac{1}{2}+\mathrm{i} x\right)\right|^{2} & =\frac{\pi}{\cosh \pi x}, \\
|\Gamma(\mathrm{i} x)|^{2} & =\frac{\pi}{x \sinh \pi x},
\end{aligned}
$$

and setting in (5.14) $m=\frac{1}{2}$, and $\hat{c}_{\frac{1}{2}}=1$, we get the normalized pseudospherical functions

$$
\hat{\mathcal{Y}}_{\lambda}^{\frac{1}{2}}(\tau, \varphi)=\frac{1}{\sqrt{2} \pi} e^{\frac{i \varphi}{2}} \frac{\cos \lambda \tau}{\sqrt{\cosh \tau}} .
$$

Therefore, in opposition to (5.25) the "vacuum vector" for $m=\frac{1}{2}$ is represented by an elementary function. Similarly, using (5.48) and the identity [20]

$$
\begin{aligned}
(-1)^{n} & \frac{\Gamma(\alpha+n) \Gamma(\gamma-\beta+n) \Gamma(\gamma)}{\Gamma(\alpha) \Gamma(\gamma-\beta) \Gamma(\gamma+n)}(1-z)^{\alpha-1}{ }_{2} F_{1}(\alpha+n, \beta ; \gamma+n ; z) \\
& =\frac{d^{n}}{d z^{n}}\left[(1-z)^{\alpha+n-1}{ }_{2} F_{1}(\alpha, \beta ; \gamma ; z)\right],
\end{aligned}
$$

we obtain from (5.15)

$$
\overline{\mathcal{Y}}_{\lambda}^{\frac{1}{2}}(\tau, \varphi)=\frac{1}{\sqrt{2} \pi} e^{\frac{i \varphi}{2}} \frac{\sin \lambda \tau}{\sqrt{\cosh \tau}} .
$$


We now study the Fock space structure of the continuous principal series representation for half-integer $m$. We begin with the functions $\mathcal{Y}_{1_{\lambda}}^{m}(\tau, \varphi)$. From (5.16a) we obtain for $m=l+\frac{1}{2}$

$$
\left|\lambda, l+\frac{1}{2}\right\rangle=\frac{1}{\prod_{r=1}^{l} \sqrt{r^{2}+\lambda^{2}}} K_{+}^{l}\left|\lambda, \frac{1}{2}\right\rangle, \quad l=1,2, \ldots
$$

Hence, taking into account (3.8) and (5.51) we find

$\mathcal{Y}_{1_{\lambda}}^{l+\frac{1}{2}}(\tau, \varphi)=\frac{(-1)^{l}}{\sqrt{2} \pi} \frac{1}{\prod_{r=1}^{l} \sqrt{r^{2}+\lambda^{2}}} e^{\mathrm{i}\left(l+\frac{1}{2} \varphi\right)} \cosh ^{l+\frac{1}{2}} \tau \frac{d^{l}}{d(\sinh \tau)^{l}} \frac{\cos \lambda \tau}{\cosh \tau}$.

Finally, using the identity

$$
\cos \lambda \tau=\frac{1}{2}\left[\left(\sqrt{1+\sinh ^{2} \tau}+\sinh \tau\right)^{\mathrm{i} \lambda}+\left(\sqrt{1+\sinh ^{2} \tau}-\sinh \tau\right)^{\mathrm{i} \lambda}\right]
$$

we obtain the formula

$\mathcal{Y}_{1_{\lambda}}^{l+\frac{1}{2}}(\tau, \varphi)=\frac{(-1)^{l}}{2 \sqrt{2} \pi} \frac{1}{\prod_{r=1}^{l} \sqrt{r^{2}+\lambda^{2}}} \mathrm{e}^{\mathrm{i}\left(l+\frac{1}{2}\right) \varphi} \cosh ^{l+\frac{1}{2}} \tau$

$\times \frac{d^{l}}{d(\sinh \tau)^{l}} \frac{1}{\sqrt{1+\sinh ^{2} \tau}}\left[\left(\sqrt{1+\sinh ^{2} \tau}+\sinh \tau\right)^{\mathrm{i} \lambda}+\left(\sqrt{1+\sinh ^{2} \tau}-\sinh \tau\right)^{\mathrm{i} \lambda}\right]$.

In view of the form of (5.57) the counterparts $\mathcal{T}_{\lambda}^{l}$ of the Legendre functions such that

$$
\mathcal{Y}_{1_{\lambda}}^{l+\frac{1}{2}}(\tau, \varphi)=\frac{1}{2 \sqrt{2} \pi} \frac{1}{\prod_{r=1}^{l} \sqrt{r^{2}+\lambda^{2}}} e^{\mathrm{i}\left(l+\frac{1}{2}\right) \varphi} \mathcal{T}_{\lambda}^{l}(\sinh \tau)
$$

are defined by

$\mathcal{T}_{\lambda}^{l}(x)=(-1)^{l}\left(1+x^{2}\right)^{\frac{1}{2}\left(l+\frac{1}{2}\right)} \frac{d^{l}}{d x^{l}} \frac{1}{\sqrt{1+x^{2}}}\left[\left(\sqrt{1+x^{2}}+x\right)^{\mathrm{i} \lambda}+\left(\sqrt{1+x^{2}}-x\right)^{\mathrm{i} \lambda}\right]$.

We now study the functions $\mathcal{Y}_{2 \lambda}^{m}(\tau, \varphi)$ for half-integer $m$. Eqs. (5.54), (3.8) and (5.53) taken together imply

$\mathcal{Y}_{2 \lambda}^{l+\frac{1}{2}}(\tau, \varphi)=\frac{(-1)^{l}}{\sqrt{2} \pi} \frac{1}{\prod_{r=1}^{l} \sqrt{r^{2}+\lambda^{2}}} e^{\mathrm{i}\left(l+\frac{1}{2} \varphi\right)} \cosh ^{l+\frac{1}{2}} \tau \frac{d^{l}}{d(\sinh \tau)^{l}} \frac{\sin \lambda \tau}{\cosh \tau}$.

An immediate consequence of the identity

$$
\sin \lambda \tau=\frac{1}{2 \mathrm{i}}\left[\left(\sqrt{1+\sinh ^{2} \tau}+\sinh \tau\right)^{\mathrm{i} \lambda}-\left(\sqrt{1+\sinh ^{2} \tau}-\sinh \tau\right)^{\mathrm{i} \lambda}\right],
$$

is the relation

$$
\begin{aligned}
& \mathcal{Y}_{2 \lambda}^{l+\frac{1}{2}}(\tau, \varphi)=\frac{(-1)^{l}}{2 \mathrm{i} \sqrt{2} \pi} \frac{1}{\prod_{r=1}^{l} \sqrt{r^{2}+\lambda^{2}}} e^{\mathrm{i}\left(l+\frac{1}{2}\right) \varphi} \cosh ^{l+\frac{1}{2}} \tau \\
& \times \frac{d^{l}}{d(\sinh \tau)^{l}} \frac{1}{\sqrt{1+\sinh ^{2} \tau}}\left[\left(\sqrt{1+\sinh ^{2} \tau}+\sinh \tau\right)^{\mathrm{i} \lambda}-\left(\sqrt{1+\sinh ^{2} \tau}-\sinh \tau\right)^{\mathrm{i} \lambda}\right] .
\end{aligned}
$$

Hence, we identify the hyperbolic counterpart $\mathcal{U}_{\lambda}^{l}$ of the Legendre functions which fulfils

$$
\mathcal{Y}_{2_{\lambda}}^{l+\frac{1}{2}}(\tau, \varphi)=\frac{1}{2 \mathrm{i} \sqrt{2} \pi} \frac{1}{\prod_{r=1}^{l} \sqrt{r^{2}+\lambda^{2}}} e^{\mathrm{i}\left(l+\frac{1}{2}\right) \varphi} \mathcal{U}_{\lambda}^{l}(\sinh \tau)
$$


such that

$\mathcal{U}_{\lambda}^{l}(x)=(-1)^{l}\left(1+x^{2}\right)^{\frac{1}{2}\left(l+\frac{1}{2}\right)} \frac{d^{l}}{d x^{l}} \frac{1}{\sqrt{1+x^{2}}}\left[\left(\sqrt{1+x^{2}}+x\right)^{\mathrm{i} \lambda}-\left(\sqrt{1+x^{2}}-x\right)^{\mathrm{i} \lambda}\right]$.

We remark that the form of the functions $\mathcal{T}_{\lambda}^{l}$ and $\mathcal{U}_{\lambda}^{l}$ suggests that they are somehow related to the Chebyshev polynomials $T_{n}(x)$ and $U_{n}(x)$.

\section{Pseudospherical functions for continuous supplementary series}

We finally discuss the pseudospherical functions for the continuous supplementary series. We first introduce the parameter $\gamma$ such that

$$
k=\gamma-\frac{1}{2}
$$

where in view of (2.14) $0<\gamma<\frac{1}{2}$. By virtue of (2.13) in this parametrization we can formally get the formulae for the supplementary series from that obtained for the continuous principal series by setting $\lambda=-\mathrm{i} \gamma$. Therefore, the even and odd spherical functions $\hat{\mathcal{Y}}_{\gamma}^{m}(\tau, \varphi)$ and $\overline{\mathcal{Y}}_{\gamma}^{m}(\tau, \varphi)$ for the continuous supplementary series obtained from (5.1) and (5.2), respectively, are given by

$\hat{\mathcal{Y}}_{\gamma}^{m}(\tau, \varphi)=e^{\mathrm{i} m \varphi} \hat{c}_{m \gamma} \cosh ^{-\left(\frac{1}{2}+\gamma\right)} \tau_{2} F_{1}\left[\frac{1}{2}\left(m+\frac{1}{2}+\gamma\right), \frac{1}{2}\left(-m+\frac{1}{2}+\gamma\right) ; \frac{1}{2} ; \operatorname{tgh}^{2} \tau\right]$,

$\overline{\mathcal{Y}}_{\gamma}^{m}(\tau, \varphi)=e^{\mathrm{i} m \varphi} \bar{c}_{m \gamma} \cosh ^{-\left(\frac{1}{2}+\gamma\right)} \tau \operatorname{tgh} \tau_{2} F_{1}\left[\frac{1}{2}\left(m+\frac{3}{2}+\gamma\right), \frac{1}{2}\left(-m+\frac{3}{2}+\gamma\right) ; \frac{3}{2} ; \operatorname{tgh}^{2} \tau\right]$.

Consider the even functions $\hat{\mathcal{Y}}_{\gamma}^{m}$. An immediate consequence of (5.5) is the relation

$$
\begin{aligned}
{ }_{2} F_{1}\left[\frac{1}{2}\left(m+\frac{1}{2}+\gamma\right), \frac{1}{2}\left(-m+\frac{1}{2}+\gamma\right) ; \frac{1}{2} ; \operatorname{tgh}^{2} \tau\right] \\
=\hat{A}_{12} F_{1}\left[\frac{1}{2}\left(m+\frac{1}{2}+\gamma\right), \frac{1}{2}\left(-m+\frac{1}{2}+\gamma\right) ; 1+\gamma ; \frac{1}{\cosh ^{2} \tau}\right] \\
\quad+\hat{A}_{2} \cosh ^{2 \gamma} \tau_{2} F_{1}\left[\frac{1}{2}\left(-m+\frac{1}{2}-\gamma\right), \frac{1}{2}\left(m+\frac{1}{2}-\gamma\right) ; 1-\gamma ; \frac{1}{\cosh ^{2} \tau}\right],
\end{aligned}
$$

where

$$
\begin{aligned}
& \hat{A}_{1}=\frac{\sqrt{\pi} \Gamma(-\gamma)}{\Gamma\left[\frac{1}{2}\left(-m+\frac{1}{2}-\gamma\right)\right] \Gamma\left[\frac{1}{2}\left(m+\frac{1}{2}-\gamma\right)\right]}, \\
& \hat{A}_{2}=\frac{\sqrt{\pi} \Gamma(\gamma)}{\Gamma\left[\frac{1}{2}\left(m+\frac{1}{2}+\gamma\right)\right] \Gamma\left[\frac{1}{2}\left(-m+\frac{1}{2}+\gamma\right)\right]} .
\end{aligned}
$$

Notice that $\hat{A}_{2}(\gamma)=\hat{A}_{1}(-\gamma)$. Proceeding analogously as with (5.1) we arrive at the following asymptotic form of $\hat{\mathcal{Y}}_{\gamma}^{m}$ :

$$
\lim _{\tau \rightarrow \infty} \hat{\mathcal{Y}}_{\gamma}^{m}(\tau, \varphi)=e^{\mathrm{i} m \varphi} \hat{c}_{m \gamma} \cosh ^{-\frac{1}{2}} \tau\left[B(\gamma) e^{-\gamma \tau}+B(-\gamma) e^{\gamma \tau}\right]
$$

where $B(\gamma) B(-\gamma)=\hat{A}_{1} \hat{A}_{2}$. In opposition to the functions (5.8) the norm of the functions (6.6) is divergent. The same result holds true for the asymptotics of the odd functions $\overline{\mathcal{Y}}_{\gamma}^{m}(\tau, \varphi)$ as well. 


\section{Acknowledgments}

This work was supported by the grant N N202 205738 from the National Centre for Research and Development.

\section{Appendix A.}

We now collect the basic relations satisfied by the functions $\mathcal{P}_{k}^{m}(x)$ given by (3.11) which are counterpart of the associated Legendre functions related to spherical harmonics. Firstly, in view of $(3.10),(2.20)$ and $(2.21) \mathcal{P}_{k}^{m}(x)$ satisfies the equation

$$
\left(1+x^{2}\right) \frac{d^{2} \mathcal{P}_{k}^{m}(x)}{d x^{2}}+2 x \frac{d \mathcal{P}_{k}^{m}(x)}{d x}+\left[-k(k+1)+\frac{m^{2}}{1+x^{2}}\right] \mathcal{P}_{k}^{m}(x)=0 .
$$

Further, an immediate consequence of differentiation of (3.11) is

$$
\left(1+x^{2}\right) \frac{d \mathcal{P}_{k}^{m}(x)}{d x}=-\sqrt{1+x^{2}} \mathcal{P}_{k}^{m+1}(x)+m x \mathcal{P}_{k}^{m}(x)
$$

Eqs. (A.1) and (A.2) taken together yield

$\mathcal{P}_{k}^{m+2}(x)-2(m+1) \frac{x}{\sqrt{1+x^{2}}} \mathcal{P}_{k}^{m+1}(x)+(m-k)(m+k+1) \mathcal{P}_{k}^{m}(x)=0$.

On reindexing $m \rightarrow m-1$ in (A.3) we can convert (A.2) into

$$
\left(1+x^{2}\right) \frac{d \mathcal{P}_{k}^{m}(x)}{d x}=(m+k)(m-k-1) \sqrt{1+x^{2}} \mathcal{P}_{k}^{m-1}(x)-m x \mathcal{P}_{k}^{m}(x) .
$$

The following identity can be obtained from (3.11) by reindexing $m \rightarrow m-1, k \rightarrow k-1$ and using the Leibniz formula on $n$-th derivative of product of functions

$2 k \mathcal{P}_{k-1}^{m}(x)=\sqrt{1+x^{2}} \mathcal{P}_{k}^{m+1}(x)-2(m-k) x \mathcal{P}_{k}^{m}(x)+\sqrt{1+x^{2}}(m-k)(m-k-1) \mathcal{P}_{k}^{m-1}(x)$. (A.

Finally, reindexing $m \rightarrow m-1$ in (A.3) and combining the resulting form of (A.3) with (A.5) we get

$$
\mathcal{P}_{k-1}^{m}(x)-x \mathcal{P}_{k}^{m}(x)=(k-m+1) \sqrt{1+x^{2}} \mathcal{P}_{k}^{m-1}(x) .
$$

We point out the remarkable similarity of the structure of above recurrences satisfied by the functions $\mathcal{P}_{k}^{m}(x)$ and recursive relations for the Legendre functions $P_{j}^{m}(x)$ connected with the spherical harmonics. For example the counterpart of (A.2) is of the form [21]

$$
\left(1-x^{2}\right) \frac{d P_{j}^{m}(x)}{d x}=-\sqrt{1-x^{2}} P_{j}^{m+1}(x)-m x P_{j}^{m}(x),
$$

and the formula corresponding to (A.6) is given by

$$
P_{j-1}^{m}(x)-x P_{j}^{m}(x)=(j-m+1) \sqrt{1-x^{2}} P_{j}^{m-1}(x) .
$$




\section{References}

[1] Gitman D M and Shelepin A L 1997 J. Phys. A: Math. Gen. 306093

[2] Gazeau J P 2010 Coherent States in Quantum Physics (New York: Wiley)

[3] Smorodinski Ya A, Shelepin A L and Shelepin L A 1992 Usp. Fiz. Nauk 16212

[4] Dane C and Verdiyev Y A 1996 J. Math. Phys. 3739

[5] Pogosyan G, Sissakian A and Winternitz P 2002 Physics of Particles and Nuclei 33 suppl. 1123

[6] Jorjadze G and Piechocki W 1999 Phys. Lett. B 461183

[7] Piechocki W 2003 Class. Quant. Grav. 202491

[8] Piechocki W 2004 Class. Quant. Grav. 21331

[9] Gazeau J P and Piechocki W 2004 J. Phys. A: Math. Gen. 376977

[10] Liu Q H, Hou J X, Xiao Y P and Li L X 2004 Internat. J. Theoret. Phys. 431011

[11] Raczka R, Limić N and Niederle J 1966 J. Math. Phys. 7 1861; Limić N, Niederle J and Raczka R 1966 J. Math. Phys. 7 2026; Limić N, Niederle J and Rączka R 1967 J. Math. Phys. 81079

[12] Strichartz R S 1973 J. Funct. Analysis 12341

[13] Molchanov V F 1976 Mat. Sb. 99139

[14] Lindblad G and Nagel B 1970 Ann. Inst. H. Poincaré, Section A 1325

[15] Wulfman C E and Levine R D 1983 Chem. Phys. Lett. 97 361; Frank A and Wolf K B 1984 Phys. Rev. Lett. 521737

[16] Messiah A 1961 Quantum Mechanics (New York: Interscience)

[17] Prudnikov A P, Brychkov Yu A and Marichev O I 2002 Integrals and Series. Vol. 1. Elementary Functions (Moscow: Fizmatlig).

[18] Brychkov Yu A 2006 Special Functions. Derivatives, Integrals, Series and Other Formulas. Handbook (Moscow: Fizmatlig)

[19] Kamke E 1959 Differentialgleichungen. Lösungsmethoden und Lösungen. Vol II. Partielle Differentialgleichungen Erster Ordnung für Eine Gesüchte Funktion (Leipzig: Akademische Verlagesellschraft)

[20] Bateman Manuscript Project 1953, edited by Erdélyi, Higher Transcendental Functions, vol. 1 (New York: McGraw-Hill)

[21] Gradshteyn I S and Ryzhik I M 2007 Tables of Integrals, Series, and Products (Amsterdam: Elsevier) 